\title{
COCOMPLETENESS OVER COVERINGS
}

\author{
RENATO BETTI \\ (Received 17 November 1983) \\ Communicated by R. H. Street
}

\begin{abstract}
For enriched categories the correct notion of limit involves indexing by a module. This paper studies the question of cocompletion for a given set of indexing modules. As well as providing a simplified treatment of cocompleteness for ordinary categories, associated sheaves and associated stacks are also included as cocompletion processes for appropriate bases. In fact the saturation of a general set of indexing modules has properties which justify our use of the term "covering" for members of the saturation.
\end{abstract}

1980 Mathematics subject classification (Amer. Math. Soc.): 18 D 20, 18 A 35, 18 F 20.

\section{Introduction}

The aim of this work is to study cocompleteness of enriched categories in terms of sets of indexing modules. Several authors (e.g. Kock [7], Wood [14], Tholen [12]) have investigated the problem of freely adjoining colimits by requiring certain stability conditions on the set of diagrams considered. As Street [10] observes, no stability condition should be necessary for the formation of the free categories. These conditions are required if one wishes to answer a more general question: how can the set of indexing modules be extended without altering the class of cocomplete categories?

In this paper we answer this question by introducing a notion of covering, closed under representables and suitable compositions, which contains as particular cases various notions of cover already introduced (Street [11], Betti and

(C) 1985 Australian Mathematical Society 0263-6115/85 $\$ A 2.00+0.00$ 
Carboni [3], Walters [13]). The free cocompletion can thus be obtained in one step, directly by the family of coverings.

Then we consider the particular case where adjoint pairs of modules are the coverings. From this point of view the paper can be regarded as a study of those properties which allow the characterization of small Cauchy-complete categories as the cocomplete ones with respect to adjoint pairs.

The base bicategory $\mathscr{B}$ is supposed to be locally small-complete and cocomplete. Moreover it is assumed that $\mathscr{B}$ is closed, i.e. it admits right Kan extensions and right liftings. Thus the composition preserves the colimits existing in the hom-categories.

For notions relative to the theory of categories enriched on a bicategory we refer to [1] and [2], where they are developed in the case of $\mathscr{B}$ locally partiallyordered, to Street [11], or to [4], where they are developed in the general case, but with slightly different notations from the present.

\section{Colimits indexed by bimodules and families of coverings}

Let $A$ be a small category, $G: A \rightarrow \mathscr{X}$ any functor and $\psi: A \nrightarrow u$ any module ( $u$ is an object of the base bicategory $\mathscr{B}$ and here it denotes also the trivial one-object category over $u$ ). We recall (Borceux and Kelly [5], Street [11]) that the $\psi$-indexed colimit $\psi * G$ of $G$ is an object of $\mathscr{X}$ (when it exists) which represents the right Kan extension

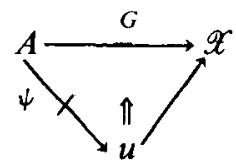

$$
\mathscr{X}(\psi * G,-) \cong \operatorname{hom}^{A}\left(\psi, G_{*}\right)
$$

By definition the colimit $\psi * G$ is characterized by a family of isomorphisms

$$
\mathscr{X}\left(\psi * G, x^{\prime}\right) \cong \mathscr{P} A\left(\psi, \mathscr{X}\left(G-, x^{\prime}\right)\right)
$$

where $\mathscr{P} A$ denotes the category whose objects over $u$ are modules $A \nrightarrow u$ and whose homs are given by right Kan extensions.

We give here a few facts, whose proofs consist of easy calculations.

Remarks. 1. When $\psi \simeq A(-, a)$ is a representable module, then $\psi * G$ exists and is (isomorphic to) $G a$.

2. $\mathscr{P} A$ classifies modules, i.e. there is a natural equivalence indicated by

$$
\mathscr{X} \stackrel{F}{\rightarrow} \mathscr{P} A
$$

where $\hat{F}(a, x)=(F x)_{a}$. 
3. $\mathscr{P} A$ is the free cocomplete category generated by $A$. The colimit of the functor $F$ indexed by the module $\psi$ can be calculated as the composite $\psi \cdot \hat{F}$, where $\hat{F}$ is obtained as in the previous remark.

4. When $\psi$ admits a left adjoint $\phi$, then $\mathscr{P} A(\psi, \alpha) \simeq \alpha \cdot \phi$.

DEFINITION. A family of coverings $J$ consists in the assignment, to each object $u$ in the base, of a set $J(u)$ of modules $A \nrightarrow u$ ( $A$ small), such that

(i) representables $A(-, a): A \nrightarrow e a$ cover;

(ii) let $\Phi: C \nrightarrow A$ be such that $\Phi(-, a)$ : $C \nrightarrow e a$ covers for each $a$, and let $\psi$ : $A \nrightarrow u$ be any covering module; then the composite $\psi \cdot \Phi: C \nrightarrow u$ covers.

A category $\mathscr{X}$ is said to be $J$-cocomplete when it admits all colimits indexed by the modules of $J$. Analogously cocontinuity relativizes to $J$-cocontinuity.

Given a family of coverings $J$, a covering module is defined to be any $\Phi: C \nrightarrow A$ which satisfies (ii) in the definition. Modules of the form $F^{*}: C \nrightarrow A$ for a functor $F: A \rightarrow C$ are always covering modules and the assignment $F \mapsto F^{*}$ amounts to a locally faithful homomorphism (which is the identity on objects) between the category of small categories and functors and the opposite category of small categories and covering modules.

The following result motivates the notion of covering module.

Lemma. Let $\mathscr{F}(u)$ be an arbitrary assignment of a set of modules $A \nrightarrow u(A$ small) to each object $u$. If the category $\mathscr{X}$ is cocomplete with respect to all the modules of $\mathscr{F}=\cup_{u} \mathscr{F}(u)$, then $\mathscr{X}$ admits also the colimits indexed by the composite modules $C \underset{\Phi}{\rightarrow} A \underset{\psi}{\rightarrow} u$, where $\psi \in \mathscr{F}(u)$ and $\mathscr{F}(-, a) \in \mathscr{F}(e a)$ for each $a$.

Proof. Given any functor $F: C \rightarrow \mathscr{X}$, the Kan extension $\operatorname{hom}^{C}\left(\Phi, F_{*}\right)$

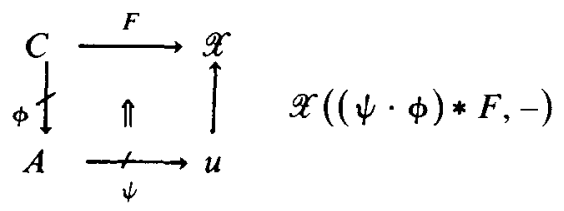

is represented by the functor $R: A \rightarrow \mathscr{X}$ defined by $R a=\phi(-, a) * F$. So we have $\operatorname{hom}^{C}\left(\Phi, F_{*}\right) \cong R_{*}$. Moreover $\operatorname{hom}^{C}\left(\psi \cdot \Phi, F_{*}\right) \cong \operatorname{hom}^{A}\left(\psi, R_{*}\right)$, and hence $(\psi \cdot \Phi) * F \cong \psi * R$, which exists by assumption.

Let us now consider any assignment $\mathscr{F}(u)$ as in the statement of the lemma, and let us adjoin representables. We denote by $J_{\mathscr{F}}$ the smallest (with respect to inclusion) family of coverings such that $\mathscr{F}(u) \subset J_{\mathscr{F}}(u)$ for each $u$. We say that $J_{\mathscr{F}}$ is generated by $\mathscr{F}$. 
THEOREM. The category $\mathscr{X}$ is $J_{\mathscr{F}}$ cocomplete if and only if it admits all colimits indexed by the modules of $\mathscr{F}$.

Proof. We construct the following sequence by transfinite induction: $\mathscr{F}_{0}(u)=$ $\mathscr{F}(u)$ and $\mathscr{F}_{\alpha+1}(u)$ is $\mathscr{F}_{\alpha}$ together with all modules of the form

$$
C \underset{\Phi}{\rightarrow} A \underset{\psi}{\leftrightarrow} u
$$

with $\psi \in \mathscr{F}_{\alpha}(u)$ and $\Phi(-, a) \in \mathscr{F}_{0}(e a)$ for each $a$. When $\alpha$ is a limit-ordinal, take $\mathscr{F}_{\alpha}(u)=\cup_{\beta<\alpha} \mathscr{F}_{\beta}(u)$.

Clearly $\mathscr{F}_{\alpha}(u) \subset J_{\mathscr{F}}(u)$ for each $\alpha$ and each $u$. Hence the sequence $\mathscr{F}_{\alpha}$ converges and $\bigcup_{\alpha} \mathscr{F}_{\alpha} \subset J_{\mathscr{F}}$. But $\bigcup_{\alpha} \mathscr{F}_{\alpha}$ is a family of coverings, and so $\bigcup_{\alpha} \mathscr{F}_{\alpha}=J_{\mathscr{F}}$.

The result now follows from the previous lemma and the induction hypothesis.

Given a family of coverings $J$, we can now consider the full subcategory $\operatorname{Cov} A$ of $\mathscr{P} A$ whose objects over $u$ are covering modules $A \nrightarrow u$. Let $i: A \rightarrow \operatorname{Cov} A$ denote the Yoneda embedding $a \mapsto A(-, a)$ which lands in $\operatorname{Cov} A$.

THEOREM. Cov $C$ is the free $J$-cocompletion of $C$.

Proof. First observe that $\operatorname{Cov} C$ classifies covering modules with domain $C$, i.e. the bijection (1) of Remark 2 restricts to the following:

$$
\frac{A \stackrel{F}{\rightarrow} \operatorname{Cov} C}{C \underset{\hat{F}}{\rightarrow} A}
$$

where $\hat{F}$ is a covering module.

Then we have that $\operatorname{Cov} C$ is $J$-cocomplete: for each functor $F: A \rightarrow \operatorname{Cov} C$, and any $\psi$ in $J$, assumption (ii) of the definition of family of coverings ensures that $\psi \cdot \hat{F}$ (i.e. the colimit of $\psi * F$, see Remark 3 ) does belong to $\operatorname{Cov} C$.

Now let us consider a $J$-cocomplete category $\mathscr{X}$ and a functor $F: C \rightarrow \mathscr{X}$. A functor $F^{\prime}: \operatorname{Cov} C \rightarrow \mathscr{X}$ is determined by $F^{\prime}(\psi)=\psi * F$, when $\psi: C \nrightarrow u$ is in $J$. It is easy to check that $F^{\prime}$ is unique (up to isomorphism) with the property $F^{\prime} \cdot i \cong F$.

REMARK. In the terminology of Kelly [7, page 183] the functor Cov amounts to "closure under $J$-colimits". The main difference here is that such closure $\operatorname{Cov} C$ is calculated on indexing modules and not within $\mathscr{P} C$.

As a corollary of the previous theorem we have the equivalence

$$
J-\operatorname{cocts}(\operatorname{Cov} C, \mathscr{X}) \simeq \operatorname{Fun}(\mathscr{X}, C)
$$

when $\mathscr{X}$ is $J$-cocomplete, as in the classical case. However the existence of right adjoints to the cocontinuous functors $\operatorname{Cov} C \rightarrow \mathscr{X}$ does not relativize to $J$ without more assumptions. 
THEOREM. Let $\mathscr{X}$ be any $J$-cocomplete category and $F: C \rightarrow \mathscr{X}$ any functor. Then $F^{\prime}: \operatorname{Cov} C \rightarrow \mathscr{X}$ admits a right adjoint if and only if the modules $F_{*}(-, x): C \rightarrow \mathrm{ex}$ cover for each $x$.

Proof. Just observe that the right adjoint of $F^{\prime}$ is given by $F_{*}(-, x)$.

\section{Examples}

1. Maximal covering. Any module $A \nrightarrow u$ covers. In this case $\operatorname{Cov} C \cong \mathscr{P} C$.

2. Cauchy-coverings. A module $\psi: A \nrightarrow u$ covers if it admits a left adjoint. In this case the $J$-cocomplete categories are the Cauchy-complete ones (see Street [11]) and $\operatorname{Cov} C$ is the Cauchy-completion.

3. Grothendieck-coverings. A topology in $\mathscr{B}$ (according to [3]) is a closure operator ( $): \mathscr{B} \rightarrow \mathscr{B}$ which is the identity on objects and which is locally left exact. The modules $\psi: A \rightarrow u$ such that the closure $\bar{\psi}$ has a left adjoint constitute a family of coverings. In this case $J$-cocomplete categories are sheaves and $\operatorname{Cov} C$ is the associated sheaf for such a topology. When $\mathscr{B}=\operatorname{Rel} C$ and the closure operator ( $)$ are constructed out of a site as in [3], then $J$-cocomplete categories are sheaves for the given site.

4. Torsors. Street [11] introduces the notion of an $A$-torsor in terms of a module $\psi: A \nrightarrow u$ which is "locally-convergent", i.e. for which there exists an arrow $f$ : $u \rightarrow v$ in a given set of "covers" such that the composite $f \cdot \psi: A \nrightarrow u \rightarrow v$ is representable. To show that torsors constitute a family of coverings it suffices to observe that the given set of "covers" is required to be a left ideal. The $J$-cocomplete categories are stacks in the sense of Street [11], and $\operatorname{Cov} C$ is the associated stack.

5. Tensor coverings. Consider the family of coverings generated by representables and by the arrows of $\mathscr{B}$ (when $\mathscr{B}$ is locally small). In this case the colimit $c * f$ of a function $c: u \rightarrow C$ (i.e. an object of $C$ over $u$ ) is exactly the tensor $c \otimes f$ and $J$-cocomplete categories are tensored categories.

6. Fiber coverings. Consider the family of coverings generated by representables and the modules $A \nrightarrow u$ where $A$ is a discrete category with just an underlying object. Then $J$-cocomplete categories are categories which admit small coproducts on the same fiber. When $\mathscr{B}$ is locally partially ordered these colimits and tensors allow one to construct all colimits.

7. Conical colimits. It is known that usual colimits can be obtained as indexed colimits (see Kelly [7], page 81). Namely, when the base $\mathscr{B}$ is Sets considered as a one-object bicategory, consider the family of coverings generated by representables and modules of the form $\phi: A \nrightarrow 1$, where 1 is the trivial one object, where $A$ 
belongs to a given set of "diagrams", and where $\phi$ is trivial $(\phi(a)$ is the one element set for each object $a$ in $A$ ).

It is easy to check that in this case the value at $x$ of the right Kan extension $\operatorname{hom}^{A}(\phi, F)$, for a functor $F: A \rightarrow \mathscr{X}$, gives the set of cocones on $F$ with vertex $x$ : Cocones $(x, F)$. So $\phi * F$, when it exists, provides a colimit in the usual sense.

In particular, the family of coverings relative to categories with small (finite) coproducts is generated in the above way by small (finite) discrete categories. Analogously, the family relative to categories which admit coequalizers is generated by the trivial module with domain in the category $0 \rightrightarrows 1$.

A somewhat different example is the following.

8. Sheaves on sites. When $(C, J)$ is a site, it gives rise to a family of coverings in $\operatorname{Rel}(C)$-Cat, in the following way: each crible $X=\left\{r_{i}: u_{i} \rightarrow u\right\}$ in $C$ determines a $\operatorname{Rel}(C)$-category (which we call again $X$ ) and a module $\psi: X \nrightarrow u$. Objects of $X$ are the arrows $r_{i}$ of the crible, the underlying of $r_{i}$ is $u_{i}$ and the hom is provided by the set of spans $(h, k)$ which commute with the maps of the crible $X$ :

$$
X\left(r_{i}, r_{j}\right)=\left\{(h, k): r_{i} \cdot h=r_{j} \cdot k\right\}
$$

The module $\psi$ is given by

$$
\psi\left(r_{i}\right)=\sup _{J} r_{j} \cdot X\left(r_{i}, r_{j}\right)
$$

where the sup is taken in the ordered set $\operatorname{Rel}(C)\left(u_{i}, u\right)$.

Let us consider the family of coverings constituted by all modules arising from covering cribles in the above way. The proof that it is a family of coverings rests on the properties of the topology $J$.

It is known (Walters [13]) that any presheaf $F$ on $C$ can be regarded as the (symmetric, skeletal, Cauchy-complete) $\operatorname{Rel}(C)$-category $L F$ having $F u$ as set of objects over $u$, and for which the hom is defined by

$$
L F(s, t)=\left\{(h, k):\left.s\right|_{h}=\left.t\right|_{k}\right\} \text {. }
$$

It is easy to check that to assign a functor $G: X \rightarrow L F$ is exactly to assign a compatible family of sections $\left\{s_{i}\right\}$ on the covering crible $X$. Thus $F$ is a sheaf exactly when, regarded as a $\operatorname{Rel}(C)$-category, $L F$ is cocomplete with respect to the induced family of coverings. It is straightforward to verify that $\psi * F$ provides a section $s$ over $u$ which restricts to $s_{i}$. The existence of $s$ is due to the fact that $\psi * F$ is a right extension; moreover, $s$ is uniquely determined because $\psi * F$ is the right Kan extension.

Besides the equivalence

$$
\operatorname{sh}_{J} C \simeq \operatorname{Rel}_{J}(C)-\text { cat }_{c c}
$$

(cc means Cauchy-complete) established in Walters [13] we have also

$$
\operatorname{sh}_{J} C \simeq J \text {-cocomp } \operatorname{Rel}(C) \text {-cat }{ }_{\mathrm{cc}} \text {. }
$$


Moreover the $J$-cocompletion process amounts to the sheafification functor.

9. Stacks. The previous example can be extended to stacks in the sense of Giraud [6]. In [4] it has been shown that

$$
\operatorname{hom}\left(C^{\mathrm{op}}, \mathrm{Cat}_{\mathrm{cc}}\right) \simeq W \text {-cat }_{\mathrm{cc}}
$$

where $W$ is the bicategory having the same objects of $C$ and arrows defined by

$$
W(u, v)=\operatorname{Sets}^{C^{\mathrm{op}}} / u \times v
$$

with the obvious composition. The 2-cells are natural transformations.

As in the previous example, from any crible $X=\left\{r_{i}: u_{i} \rightarrow u\right\}$ in $C$, we get a $W$-category $X$ and a module $\psi: X \nrightarrow u$. The objects of $X$ are given by the arrows $r_{i}$, and $X\left(r_{i}, r_{j}\right)$ is the span

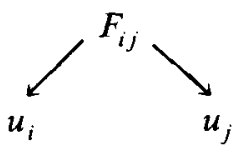

where $F_{i j}$ is the presheaf $F_{i j}(v)=\left\{(h, k): r_{i} \cdot h=r_{j} \cdot k\right\}$.

The module $\psi$ is thus defined by $\psi\left(r_{i}\right)=r_{i}$. Let us consider again the family of coverings in $W$-cat induced by the given topology. With calculations very similar to those of the previous example we have the biequivalence

$$
\left(\text { stack }_{J} C\right)_{\mathrm{cc}} \simeq J \text {-cocomp } W \text {-cat } \text { cc }_{\text {. }} \text {. }
$$

10. Discrete coverings. As a last example we can consider the minimal one: just representables cover. Any category is cocomplete with respect to this family of coverings (see Remark 1 in the previous section).

\section{On cocompleteness of small categories}

We recall that a small category $X$ is Cauchy-complete (Lawvere [9]) when each adjoint pair of modules $u \underset{\psi}{\stackrel{\phi}{\rightleftarrows}} X(\phi \dashv \psi)$ is representable. Analogously (Street [11]), $X$ is a stack when each torsor is representable. We deal here with the general case.

THEOREM. A small category $X$ is $J$-cocomplete if and only if the embedding

$$
X \underset{i}{\rightarrow} \operatorname{Cov} X
$$

has a reflection $j$.

Proof. Suppose $X$ is $J$-cocomplete. Then

$\operatorname{Cov} X\left(\psi, x_{*}\right) \cong \operatorname{Cov} X(\psi, X(-, x)) \cong X(\psi * 1, x)$ 
where $\psi$ is any covering and 1 denotes the identity functor of $X$. Thus $j(\psi)=\psi * 1$ provides a left adjoint to $i$. Moreover $j(X(-, x)) \cong x$, so $j$ is a reflection.

Conversely, suppose $j$ is given. Take any functor $F: A \rightarrow X$ and any covering $\phi$ : $A \nrightarrow u$. The module $\phi \cdot F^{*}$ is again a covering, so we have the natural bijections

$$
X\left(j\left(\phi \cdot F^{*}\right), x\right) \cong \operatorname{Cov} X\left(\phi \cdot F^{*}, X(-, x)\right) .
$$

Now it is easy to check that $j\left(\phi \cdot F^{*}\right) \cong \phi * F$ is the $\phi$-indexed colimit of $F$.

We are now in a position to compare a family of coverings $J$ with the Cauchy coverings. We say that $J$ is less fine than the Cauchy-covering if each $J$-cover is also a Cauchy cover, i.e. it admits a left adjoint.

THEOREM. Suppose $J$ is less fine than the Cauchy covering. Then a small category $X$ is $J$-cocomplete if and only if any $J$-covering $X \nrightarrow u$ is representable.

Proof. Suppose that any $J$-covering $\psi: X \nrightarrow u$ is representable. Given any functor $F: A \rightarrow X$ and any $\phi: A \rightarrow u$ in $J(u)$, consider $\phi \cdot F^{*}$. By assumption $\phi \cdot F^{*} \cong X\left(-, x_{0}\right)$ for a certain object $x_{0}$. Thus $\phi \cdot F^{*}$ has a left adjoint $X\left(x_{0},-\right)$. It is easy to check that a left adjoint of $\phi \cdot F^{*}$ must be isomorphic to $\operatorname{hom}^{A}\left(\phi, F^{*}\right)$. Indeed it is true that

$$
\operatorname{hom}^{A}\left(\phi, F^{*}\right) \cong \operatorname{hom}^{X}\left(\phi \cdot F^{*}, 1\right)
$$

as is proved by the following calculation (where $\beta$ denotes an arbitrary module $u \nrightarrow X)$ :

$$
\begin{array}{ll}
\frac{B \cdot \phi \cdot F^{*} \rightarrow 1}{\beta \cdot \phi \rightarrow F_{*}} & \text { (adjunction } \left.F_{*} \dashv F^{*}\right) \\
\beta \rightarrow \operatorname{hom}^{A}\left(\phi, F_{*}\right) & \text { (Kan extension) }
\end{array}
$$

Conversely, suppose $X$ is $J$-cocomplete. Then a covering $\psi: X \nrightarrow u$ provides an object $x_{0} \cong \psi * 1$, i.e.

$$
\operatorname{hom}^{X}(\psi, 1) \cong X\left(x_{0},-\right) .
$$

So $\operatorname{hom}^{X}(\psi, 1)$ admits $X\left(-, x_{0}\right)$ as a right adjoint, i.e. $\psi \cong X\left(-, x_{0}\right)$.

\section{Acknowledgement}

I wish to express my gratitude to Ross Street for encouragement and suggestions. 


\section{References}

[1] R. Betti, 'Bicategorie di base', Quad. 2/S (II), Ist. Mat. Univ. Milano (1981).

[2] R. Betti, 'Alcune proprietà delle categorie basate su una bicategoria', Quad. 28/S (II), Ist. Mat. Univ. Milano (1982).

[3] R. Betti and A. Carboni, 'Notion of topology for bicategories', Cahiers Topologie Géom. Differentielle 24 (1983), 19-22.

[4] R. Betti, A. Carboni, R. H. Street and R. F. C. Walters, 'Variation through enrichment', J. Pure Appl. Algebra 29 (1983), 109-127.

[5] F. Borceux and G. M. Kelly, 'A notion of limit for enriched categories', Bull. Austral. Math. Soc. 12 (1975), 49-72.

[6] J. Giraud, Cohomologie non abélienne (Springer, 1971).

[7] G. M. Kelly, Basic concepts of enriched category theory (Cambridge Univ. Press, 1982).

[8] A. Kock, 'Limits monad in categories', Aarhus Univ. Matematisk Preprint No. 6, (1967/68).

[9] F. W. Lawvere, 'Metric spaces, generalized logic, and closed categories', Rend. Sem. Mat. Fis. Milano 43 (1973), 135-166.

[10] R. H. Street, 'The comprehensive construction of free colimits', Macquarie Math. Reports, May 1979.

[11] R. H. Street, 'Enriched categories and cohomology', Quaestiones Math. 6 (1983), 265-283.

[12] W. Tholen, 'Completions of categories and shape theory', Seminarberichte No. 12, FernUniversität, pp. 125-142 (1982).

[13] R. F. C. Walters, 'Sheaves on sites as Cauchy-complete categories', J. Pure Appl. Algebra 24 (1982), 95-102.

[14] R. J. Wood, 'Free colimits', J. Pure Appl. Algebra 10 (1978), 73-80.

\section{Dipartimento di Matematica}

Università di Milano

via C. Saldini, 50

20133 Milano

Italy 